\title{
Structures narratives et congruence cognitive : cas du summary lead et de la pyramide inversée
}

\author{
Bertrand Labasse
}

Résumé

Le summary lead $(5 \mathrm{~W}+\mathrm{H})$ et la construction dite en pyramide inversée sont des préceptes dominants dans le champ de la rédaction. Leur validité est examinée ici dans une triple perspective, historique (quelle origine ?), didactique (quel apport en formation ?) et technique (quelle efficacité communicationnelle ?). Cette analyse conduit à relever de profondes incohérences, mais aussi à s'interroger sur le lien entre l'état de ce type de connaissances - problème réputé seulement instrumental - et des questions plus vastes tels que le désintérêt du public pour les informations complexes.

Mots clés: communication, journalisme, normes discursives, construction, réception, cognition

\begin{abstract}
The summary lead $(5 \mathrm{~W}+1 \mathrm{H})$ and the inverted pyramid are dominant precepts in the area of writing. Their validity is examined here in three ways, historical (what origin?), didactic (what benefits for training?) and technical (what communicational efficiencies?). This analysis reveals deep incoherencies while also enabling a consideration of the link between the state of this type of knowledge - a problem reputed to be merely instrumental - and broader questions such as the lack of public interest in complex information.
\end{abstract}

Keywords: communication, journalism, discursive norms, construction, reception, cognition

\section{Introduction}

L'examen approfondi des connaissances et préceptes actuels en matière de rédaction constitue un préréquis à tout développement de ce domaine. Dans cet article, nous nous proposons d'étudier deux normes de rédaction particulièrement répandues, considérées comme deux aspects d'une même logique de composition visant à accroître le rendement informatif des textes. En examinant successivement leur 
construction historique, leur portée didactique et leur validité opératoire, nous montrerons que ces préceptes, s'ils constituent bien des notions centrales dans ce domaine, sont extrêmement fragiles, que ce soit en tant que " savoirs », en tant qu'objets d'enseignement et même en tant que techniques visant à accroître l'efficacité discursive.

\section{Un élément d'expertise appliquée : le principe de préséance de l'essentiel}

Le principe de préséance de l'essentiel est l'un des universaux majeurs de la rédaction appliquée ${ }^{1}$, c'est-à-dire l'une des rares doctrines qui soit très largement répandue dans les cours et les manuels de rédaction, et dont les contenus soient relativement formalisés. Si le principe de fond - " dire d'abord l'important » - est plutôt général, son développement associe deux points assez bien définis : le lead en 5W (ou summary lead) et la construction en pyramide inversée. Le fait que ces deux appellations ne soient pas, en elles-mêmes, parlantes pour un néophyte suggère qu'il s'agit bien d'éléments d'expertise, susceptibles d'être enseignés : elles tiennent simultanément du déclaratif (savoir) et du procédural (savoir-faire).

Rappelons-en la substance. Le lead en $5 \mathrm{~W}$ (ou $5 \mathrm{~W}+\mathrm{H}$ ) consiste à commencer un texte par son information essentielle, laquelle doit autant que possible répondre à cinq questions : Qui ? (a fait) Quoi ? Quand ? Où ? Pourquoi ? Comment ? Soit, en anglais : Who ? (did) What ? When ? Where ? Why ? How ?. La structuration en pyramide inversée repose sur le même principe - l'important d'abord - prolongé cette fois à la totalité du texte : celui-ci comporte d'abord l'information essentielle, telle que définie précédemment, puis les éléments d'information accessoires, présentés par ordre décroissant d'importance.

Si ces deux outils ne sont pas toujours associés dans les manuels ou les cours, ils ont connu, ensemble ou séparément, un succès qui ne se dément pas. Ils sont fondamentalement attachés à l'écriture de presse : "Qui a fait Quoi, Où, Quand et Pourquoi ? Ce sont les $5 \mathrm{~W}$ que l'on enseigne depuis la nuit des temps (modernes) dans toutes les écoles de journalisme du monde... » (Antoine et al., 1995, p.16). D'un auteur à l'autre, les différences portent surtout sur leur caractère absolu ou non ${ }^{2}$. Plusieurs suggèrent, implicitement ou explicitement, que ce moule est incontournable :

1 La rédaction appliquée désigne ici toutes les formes de rédaction post-scolaires et non littéraires liées à une activité, tant à titre principal (rédaction professionnelle ; par exemple rédaction journalistique) que dans le cadre d'une autre fonction (rédaction fonctionnelle ; par ex. rédaction d'affaires, scientifique, etc.)

2 Bien que certains auteurs les réfutent globalement. 
Vous rédigez d'abord l'essentiel des faits : Qui ? Où ? Quand? Pourquoi ? Comment ? (...) Cette expérience vous enseignerait que tout peut se condenser, elle vous apprendrait aussi comment se bâtit un article : d'abord une phrase qui résume l'essentiel, puis des développements successifs, par ordre d'intérêt décroissant. (Florio, 1984, pp.72-73) ${ }^{3}$

L'écriture journalistique ne peut connaître qu'un seul plan, celui qui valorise l'information centrale. (...) Le premier paragraphe de tout article doit dire le cœur de l'affaire. La suite les développe par ordre d'importance décroissante. Sans doute, les modalités de cette écriture-là sont très variées (...) Mais la construction est invariable. C'est le plan réputé " en pyramide inversée. » (Mouriquand, 1997, p. 48$)^{4}$

Comme pour mieux exprimer le caractère sacré des $5 \mathrm{~W}+\mathrm{H}$, Hartman (1968) les figure sous la forme d'un temple grec, dont le fronton porte l'inscription « ELEMENTS OF $L E A D$ » et dont chacune des six colonnes représente l'une des questions (p. 91).

Mais d'autres voient plutôt dans ces règles une norme par défaut, qui peut admettre des exceptions, essentiellement pour certains articles longs de type magazine (feature) : " just as Five W leads are inadequate for depht stories, so is the inverted pyramid." (Lorenz \& Vivian, 1996, p.98). Pour ne pas y renoncer trop vite, même lorsque le sujet doit aborder plusieurs thèmes, Ross (1994) a proposé une solution sophistiquée : "On pourrait parler de pyramide multiple : quelques petites pyramides (schématisant des divisions du texte) s'emboîtent pour en former une grande (schématisant l'ensemble de la structure du texte). » (p. 62)

II convient cependant de noter que, tandis que ces préceptes maintiennent leur domination dans les manuels et les enseignements, il peut arriver qu'ils soient remis en question avec vigueur par des praticiens (ou praticiens-enseignants) expérimentés :

In journalism programs students work primarily with the simplest, most formulaic forms of writing - forms most of their professors haven't used for years and, if truth be told, have no great desire to use again. (Stephens, 2000)

Comme on l'a dit, ces outils se sont développés bien au-delà de la rédaction journalistique pour se disséminer dans les différents domaines de la rédaction fonctionnelle. Cette influence doit probablement beaucoup à Gunning (1968), l'un des auteurs dominants en la matière : "I have run across men in business who thought this

3 L'omission assez étrange de la question centrale « Quoi ? ", sans laquelle les autres sont un peu surréalistes, est probablement une coquille, l'auteur étant un enseignant et un praticien chevronné.

4 En une contradiction fascinante mais significative, le même auteur affirmait peu avant que «La construction des écrits journalistiques, à l'inverse d’autres, ignore les moules.» (p. 32). 
was the way to write report : Feed out details gradually (...) But this is a poor way to organize a piece of writing that is chiefly information. (...) when your chief aim is to hand on information, the inverted pyramid form is best. » (p.122). De fait, le principe de préséance de l'essentiel occupe aujourd'hui une place importante dans la rédaction en milieu de travail : "Pour un écrit professionnel [sic], une lettre officielle, une note de service, répondre strictement à des questions simples : Qui ? Quoi ? Quand ? Où ? Comment ? Pourquoi ? Pour qui ? " (Peyron, 1997, p.68) ; "Ricordiamo la regola giornalistica delle cinque Wh- : chi, che cosa, quando, dove, perché (dall'iniziale dlle parole inglesi correspondenti : who 'chi?', what 'che cosa?', when 'quando ?' where 'dove ?', why 'perché?'). „ (Bruni \& Alfieri, 1997, p.37). Même la rédaction scientifique, au-delà de la sempiternelle description du plan IMRAD, s'est laissée gagner par ce modèle : "L'introduction doit être courte (...) L'essentiel doit être dit dès les premières phrases. La fameuse accroche [sic] des journalistes et des publicitaires est, en effet, capitale pour appeler et retenir le lecteur. » (Bénichoux, 1985, p.62). On peut ainsi retrouver ces idées dans les conseils donnés aux agronomes tropicaux par la revue d'un organisme de développement international (« Les modules de formation en communication écrite citent invariablement ces règles inspirées des méthodes journalistiques : (...) hiérarchiser l'information et respecter la règle des QQQOPC. Un message concis répond en effet à six questions (...)» CTA, 1999) de même que dans les suggestions que prodigue aux ingénieurs la revue d'une agence nationale de veille technologique ("La pyramide inversée : hiérarchiser pour structurer » ADIT, 1999). Enfin, la méthode connaît aujourd'hui un succès considérable chez les concepteurs de sites internet: "Une page Web n'est pas un document papier ! La lecture écran est $25 \%$ plus lente que la lecture papier : on ne lit pas à l'écran, on scanne. D'où l'importance à accorder (...) A l'efficacité d'une écriture en pyramide inversée : donner en premier lieu l'idée principale (...) et ne détailler qu'ensuite. » (Carrière \& Schreiber, 2000, p. 2).

Toutefois, si la pyramide inversée et les $5 \mathrm{~W}$ méritent qu'on s'y arrête, c'est moins pour leur poids global dans le champ de la rédaction que pour ce qu'ils révèlent de celui-ci. $A$ travers cet exemple, on constate d'abord que le journalisme exerce une ascendance inattendue - une expertise - sur l'ensemble du champ de la rédaction appliquée. Ce magistère, est, du reste, très souvent invoqué : "Les journalistes peuvent-ils constituer de bons exemples pour nous, écrivants d'entreprise ? Mais bien sûr.» (Berrou, 1991, p. 121) " My general advice for curing many of the problems that tend to recur in science writing is really quite simple : think of yourself as a reporter, as well as a scientist. You can learn something from journalists when you write the formal science report.» (Porush, 1995, p. 19). "Les journalistes ont beaucoup à nous apprendre à ce niveau » (ADIT, 1999). 
Sans débattre ici de l'ensemble des apports conceptuels du journalisme, il convient donc d'examiner avec attention les facteurs qui ont érigé la pyramide et des $5 \mathrm{~W}$ en « savoirs de référence ».

\section{Une genèse discutable}

Symboliquement, ces notions semblent représenter la transition entre une forme d'écriture ancienne, alambiquée et littéraire, et celle des " temps modernes », où l'on sait aller à l'essentiel (voir supra l'extrait d'Antoine et al.). II n'est pas indifférent de noter qu'elles sont attachées aux U.S.A., pays dont l'image est traditionnellement associée à celle d'efficacité productive. Esquissant les contours du premier vrai programme d'enseignement du journalisme d'Espagne, Graña-Gonzáles (1930) note ainsi : "Los yanquis han llegado también a un grado de perfección que debemos imitar. El lead ${ }^{5}$ (...) es un artificio técnico que prontó serà adoptado por los buenos periódicos européos. » (p. 157). De fait, contrairement à d'autres notions, qui semblent flotter dans ce domaine, venues d'on ne sait où et d'on ne sait qui, elles ont une origine claire et on ne peut plus « moderne », puisqu'elle coïncideraient avec l'essor de la civilisation industrielle et celui des « nouvelles technologies de l'information », en l'occurrence le télégraphe. L'histoire est connue :

Ce sont les défectuosités du télégraphe, en même temps que le coût de son utilisation, qui apporteront ses premières règles au journalisme américain. Ce sont elles qui imposent aux correspondants de guerre la grande concision de leur expression. (...) Ainsi, les premières recommandations aux journalistes américains sont nées des hasards de l'histoire ou des nécessités de la technique : le procédé de la pyramide inversée, qui impose la synthèse avant la présentation des détails ; le respect des cinq interrogations de l'amorce, les 5 "W» - Who, What, When, Where, Why - (Qui ? Quoi ? Quand ? Où ? et pourquoi ?) enfin la recommandation d'un style à la fois dépouillé et impersonnel. (Balle, 1987, pp. 24-25)

Cette généalogie est même reprise par un des plus farouches contempteurs de ces procédés, Flesch (1949), qui souligne que " the typical news lead is a Civil War relic. In those days, the telegraph wasn't too reliable and war correspondent invented (...) " (p.63). L'histoire de cette "invention » est séduisante, mais elle est un peu courte : l'idée générale d'aller au fait, sans fioritures, remonte au moins à Homère ${ }^{6}$ (lliade) et le

5 Le terme « lead » est en italique dans l’original, avec une note de renvoi : « Pronúnciese liid. »

6 Ainsi que le souligne plus tard Horace qui, dans un vers célèbre de son Art poétique (ou Epître aux Pisons), rappelle qu'Homère plonge son lecteur « in media res» (au milieu des choses). Pour contribuer, même très approximativement, à l'histoire des techniques rédactionnelles, aujourd'hui si lacunaire, on précisera qu'Horace est, de facto, l'un des principaux théoriciens de notre discipline. Son Art poétique a directement inspiré celui de Boileau (qui l'a traduit) et ce dernier est l'un des deux auteurs les plus cité dans notre champ. Sur 50 ouvrages (12 anglophones 
« questionnement standard » à Aristote (Ethique 3:1). Le principe général de la pyramide est notamment discuté à l'époque de Quintilien (qui, pour sa part, le rejette ${ }^{7}$ ) : "Les arguments les plus puissants doivent-ils être placés au commencement, pour s'emparer des esprits, ou à la fin, pour laisser les auditeurs sous cette impression, ou partie au commencement et partie à la fin (...) On suivra l'ordre qu'exige la cause, à une exception près : il ne faut pas une progression descendante du fort au faible. " (op. cit., Livre $V$, trad Bornecque p. 231). Le même rappelle par ailleurs les circonstances à traiter dans la narration : " personne, cause, lieu, temps, instrument, occasion » (id., p. 61). De St Thomas d'Aquin (Somme théologique,1266, la lla, Qu.7) jusqu'à Goscinny \& Uderzo (La serpe d'or,1962, p.43) en passant, dans des registres bien différents, par Kipling (1902) $)^{8}$ ou Lasswell $(1948)^{9}$, le recours au célèbre questionnement de la tradition rhétorique - Quis, Quid, Ubi, Quibus auxiliis, Cur, Quomodo, Quando? - est décidément trop constant au fil du temps pour être attribué de but en blanc au télégraphe. Mais seul un très petit nombre d'auteur paraît s'en aviser.

et 38 francophones), il est cité 14 fois, soit plus que Flesch ou Gunning (seul Richaudeau parvient à le dépasser). Bref une bonne partie des nos préceptes favoris, tels que l'importance de la méta-cognition (que tend aujourd'hui à confirmer les recherches en sciences cognitives) nous viennent de Boileau (1636:1711) transposant Horace (-65: -4) lequel invoque à son tour Homère (vers -800).

7 Perelman (1997) rappelle cependant que les ordres croissant et décroissants étaient également envisagés dans la rhétorique antique, en fonction de la cause, même si l'ordre nestorien (le plus important au début et la fin, le reste au centre) était généralement préconisé (p. 163).

8 A la fin de son conte The Elephant's Child (in Just So Stories, 1902), consacré aux dangers de la curiosité, se trouve ce quatrain : " I keep six honest serving-men // (They taught me all I knew) // Their names are What and Why and When // And How and Where and Who. »

9 C'est pour synthétiser les approches usuelles des processus de communication de masse que Lasswell, dans un article fameux, les résumera par la formule «Who // Says What // In Which Channel // To Whom // With What Effect? " (capitales selon l'original ; les doubles fractions correspondent à un retour à la ligne), son propos étant de regretter que les recherches se focalisent sur l'un ou l'autre de ces points, auxquels il oppose une approche plus globale. Toutefois, Lasswell ne se référant pas au questionnement classique, on ne peut dire si ce dernier l'a inspiré ou non 

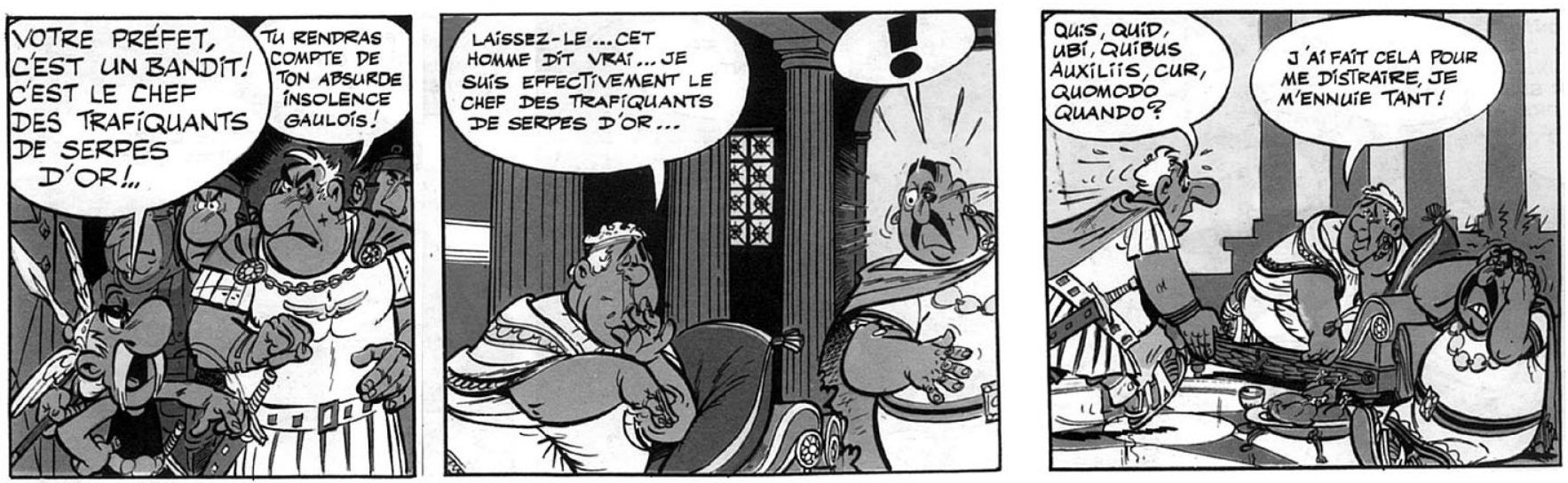

Fig. 1 : Persistance historique de l'interrogation circonstancielle (case 3, en haut à G. $)^{10}$

Quoi qu'il en soit, c'est surtout la question de son application effective qui importe. Et c'est surtout là que l'histoire collective de ces notions semble lacunaire : l'examen d'une vingtaine de quotidiens américains entre 1860 et 1910 (Errico et al., 1997) montre en effet que le taux d'articles utilisant vraiment le lead en $5 \mathrm{~W}$ reste inférieur à $1 \%$ pendant presque tout le XIXe siècle, n'atteignant 1,14\% qu'en 1895 (trente ans après la guerre civile) : les messages de ce type étaient sans doute destinés à être rewrités, et non imprimés en l'état. Un autre auteur qui a travaillé sur cette période (Mindich, 1998) suggère même que ce modèle pourrait avoir été développé, non par les journaux euxmêmes mais horresco referens, par les censeurs du ministère de la guerre. Quoi qu'il en soit, l'histoire des $5 \mathrm{~W}$ nous paraît pouvoir s'interpréter non comme une trouvaille soudaine, mais comme celle d'une confusion progressive, qui a insensiblement transformé ce qui est fondamentalement une technique de synthèse préalable de l'information - à l'origine pour structurer ses idées et plus tard pour transmettre des données essentielles - en une technique d'exposition directe de cette information.

\section{Des notions didactiquement confuses}

Un autre aspect essentiel de ces notions, nous l'avons dit, est leur valeur déclarative, donc didactique. Parce qu'elles sont désignées par des termes spécifiques, et qu'elles sont collectivement formalisées (même si l'on peut rencontrer à leur propos de nombreuses variations au hasard des auteurs) elles constituent quelque chose qui peut s'écrire ou se dire, et non de simples habiletés à conférer par la pratique. Mais ces

10 @ Editions Albert René (nota : La séquence présentée ici est remontée : l'original comporte une case de plus, non pertinente ici) 
" connaissances à enseigner » sont-elles didactiquement pertinentes ? Un premier doute peut provenir de l'apport cognitif des $5 \mathrm{~W}$ pour le rédacteur aux prises avec le matériel qu'il a à traiter. Sans s'attarder sur le fait, évident, que réduire le réel à une trame où quelqu'un a immanquablement fait quelque chose n'est souvent possible qu'au prix d'un élargissement considérable des notions d'agent et d'action (par ex. "l'inflation a augmenté... ») et paraît fort difficile à appliquer dans bien des cas, on notera que la détermination réciproque de l'agent et de l'action est généralement très délicate. Ainsi une forme telle que [Qui ? :] Le laboratoire... [Quoi ? :] ... a connu des incidents, paraît-elle informativement équivalente à [Qui ? :] Des incidents... [Quoi ? :] ... ont affecté le laboratoire. II ne s'agit évidemment là que de permutations assez vaines (Belle marquise vos yeux... Vos yeux, belle marquise... etc.) mais c'est justement à ce type d'impasse que peut conduire le questionnement $5 \mathrm{~W}$ s'il est imprudemment érigé en dogme. Ainsi peut-on se demander si, au lieu de réduire la charge cognitive du rédacteur novice, cet « élément d'expertise » ne contribue pas à l'accroître en l'orientant vers un dédale réflexif (qui devient inextricable lorsqu'un sujet concerne de nombreux agents et actions). Paradoxalement, dans bien des cas, le désir de satisfaire à ce questionnement pourra même conduire à focaliser le sujet sur un élément qui s'y prête, mais qui n'est pas l'information essentielle : la lettre de la règle se retourne dans ce cas contre l'esprit de la règle.

La notion de pyramide inversée pose, pour sa part, un problème didactique d'un autre genre. L'utilisation d'un terme géométrique ou architectural puissamment évocateur (pyramide) constitue une façon presque métaphorique de concevoir le texte. Dès lors, la question est évidemment de savoir si la représentation activée correspond à l'idée qu'elle est censée supporter. A quoi peut donc bien ressembler un texte conçu comme une pyramide inversée ? La confrontation des idées que peuvent s'en faire divers auteurs est révélatrice des limites de cette métaphorisation. 


\section{Fig. 2 : Quelques représentations du modèle pyramidal}

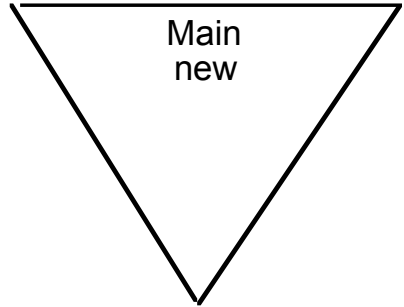

LORENZ et al. 1996 p.95

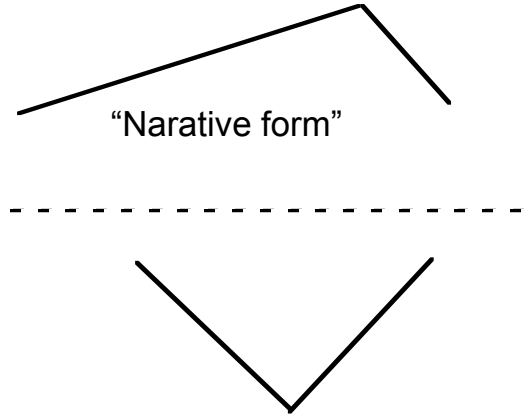

"Inverted pyramid form"

GUNNING

1968 pp.121-122

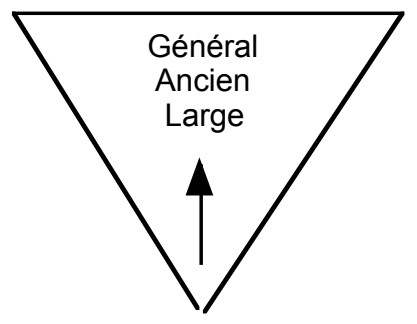

Particulier

Récent

Précis

FAURE

1998 p.109

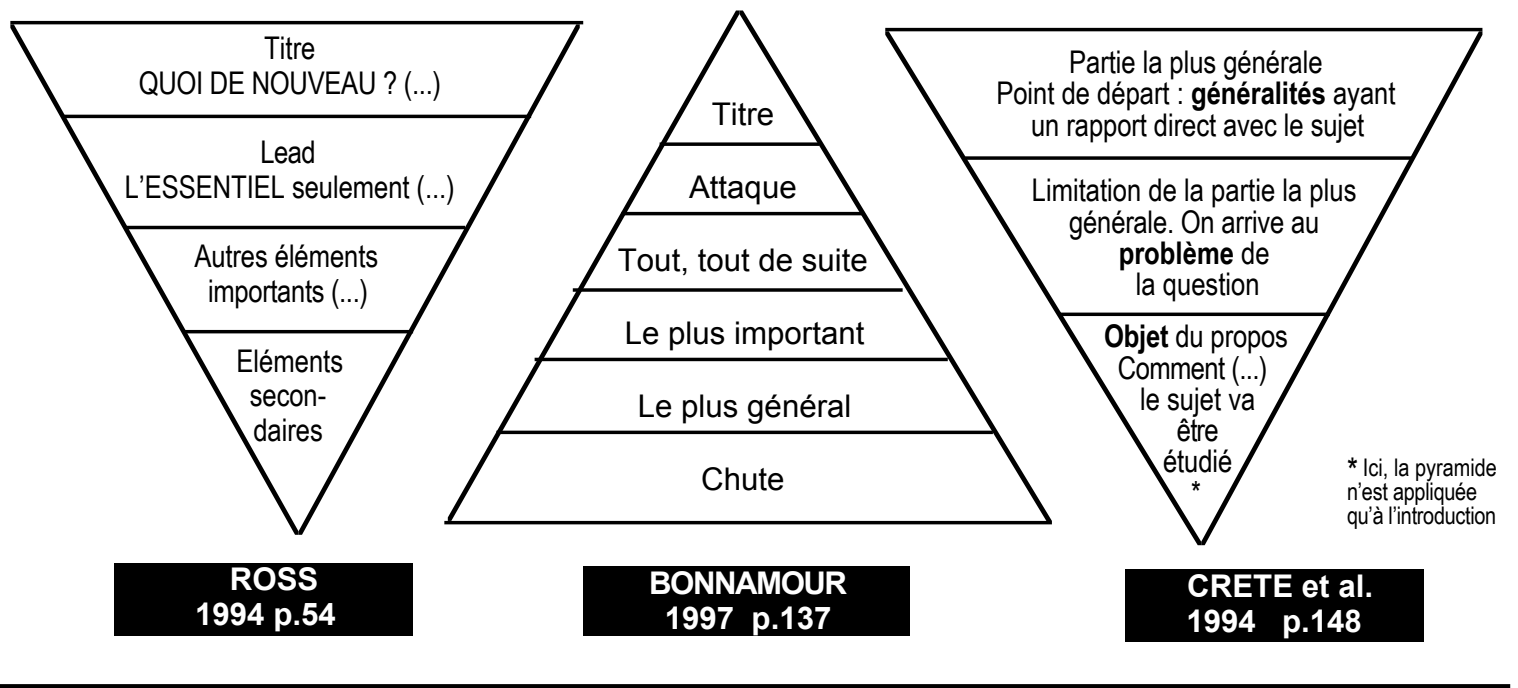

On constate sans peine que ces exemples ne sont pas compatibles entre eux, et sont même opposés sur un point majeur : le « haut » de la pyramide, donc le début du texte, correspond-il à l'information principale de ce texte, ou, au contraire, à des généralités préliminaires ? En principe, la réponse est sans équivoque : le début du texte est bien censé être la partie la plus importante de celui-ci, comment l'indiquent Gunning, Lorenz \& Vivian et Ross. Mais les inversions (variations ou contresens) que l'on remarque chez 
les autres illustrent clairement l'inadéquation cognitive de ce modèle : la métaphore géométrique suggère un critère dimensionnel (le sommet doit être plus «large » que la base) alors qu'elle ne porte que sur un critère qualitatif (le sommet doit être plus « important » que la base). Ce point est d'autant plus notable que la pyramide inversée est le seul schéma qui soit largement répandu dans le champ de la rédaction appliquée, où les discours sont essentiellement textuels. On mesure cette difficulté en constatant que Gunning rapproche deux graphismes qui ne présentent en réalité aucune cohérence. Dans ces deux figures, le critère est bien qualitatif (importance), mais le premier, qui représente les textes classiques, progressant jusqu'à un paroxysme avant de retomber vers leur conclusion, doit se lire de la gauche vers la droite (la hauteur correspondant à l' « importance " croissante des éléments), tandis que le second, qui est le modèle normal de la pyramide inversée, se lit du haut (début de l'article) vers le bas (fin), la largeur correspondant à l'« importance » des éléments. Comme le remarque justement Ross (seule à paraître s'en inquiéter) "Malgré son utilité (...) l'image de la pyramide risque de prêter à confusion. » (p.55). En l'occurrence, le « risque » paraît une certitude : le fait que les auteurs eux-mêmes puissent s'y perdre montre que le seul schéma à vocation heuristique de notre champ est non seulement peu adéquat mais même dommageable, puisque, loin de faciliter la construction d'une représentation correcte, il tend à favoriser une mauvaise compréhension.

\section{Une efficacité très variable}

La question la plus importante que posent les $5 \mathrm{~W}$ et la pyramide inversée demeure celle de leur efficacité. S'il s'agit bien, toute considération historique mise à part, de notions résolument modernes, c'est parce qu'elles ne cherchent pas leur justification dans des considérations esthétiques, des normes institutionnalisées ou dans une meilleure adéquation au sujet traité, mais dans une volonté de rendement, d'efficacité informative. Celle-ci est d'autant plus marquée qu'elle concerne simultanément les trois degrés de l'interaction textuelle :

- Le rédacteur. Le questionnement permet de rassembler ses idées, de vérifier que l'on dispose de tous les éléments nécessaires, d'éliminer l'inutile, voire de stimuler son inspiration. Beaucoup d'auteurs s'en tiennent, du reste, à cette utilisation : "Posez-vous ces questions. Elles servent d'ouvre-boîte mental. » (Barras, 1978, p. 38)

- Le texte lui-même. Le document ainsi rationalisé devient aisément synthétisable, et surtout redimensionnable en fonction des besoins et de la place disponible. C'est la raison pour laquelle cette structuration constitue une norme dans toutes les agences de presse télégraphiques : "sur la dépêche de 300 lignes (...) un journal pourra, selon ses choix propres, offrir la totalité à ses lecteurs, se contenter de 200 lignes, des 80 
premières ou du seul premier paragraphe (...) " (Hervouet, 1979 p. 73). Le même principe vaut pour des documents d'autres sources, tels que les communiqués de presse (qui visent à être partiellement ou totalement reproduits à l'identique) ou certains textes techniques particuliers.

- Le lecteur. La raison la plus fréquemment invoquée en faveur du principe même de la préséance de l'essentiel (en général) est qu'il est le plus à même de répondre aux attentes du destinataire : "Le lecteur est une personne pressée » (Boclé \& Patriot, 1997 p. 60), qui, ainsi, "entre directement dans le vif du sujet. » (Collectif, 1987, p. 150). En outre, cette préséance, quand elle s'étend à toute la structure du texte (pyramide inversée) favorise le redimensionnement de celui-ci par son destinataire lui-même puisqu'elle " permet à l'auditeur ou au lecteur de s'arrêter lorsqu'il a toute l'information qu'il désire. » (Gunning, 1968, p. 189). II s'agit donc d'un " plan psychologique » (Martin-Lagardette, 1994, p. 69).

En termes strictement matériels (longueur du texte) l'efficacité de la construction rétropyramidale ne fait aucun doute : on voit mal, en particulier, comment les agences pourraient remplir leur fonction sans y recourir. Mais ce type de nécessité reste très spécifique ; dans leur grande majorité les textes n'ont pas pour vocation essentielle d'être retaillés à volonté. Pour le reste, l'argument dominant - celui des goûts des lecteurs - repose sur une vision assez partielle de leurs attentes. Deux points sont en sa faveur. D'une part, nul ne prend plaisir à parcourir un délayage interminable, d'autre part, de nombreuses études ont montré que les lecteurs n'ont pas le temps matériel de parcourir toutes les informations qui s'offrent à eux. Mais d'un autre côté, supposer que le seul contenu de l'information est ce qui en fait la valeur (d'où l'idée de l'exposer au plus vite) est l'une des plus grandes erreurs que puisse commettre un rédacteur. Elle présuppose en effet de ses destinataires un appétit de savoir, spontané et plus ou moins universel, qu'il ne s'agirait que de satisfaire en fournissant les données correspondantes. Or, la très grande majorité des informations sont loin de bénéficier d'un tel désir. La surabondance d'informations, qui a instinctivement induit la question (passive) "Comment permettre au lecteur d'accéder vite à ce qui l'intéresse ? ", amène également une question très différente (active) « Comment amener le lecteur à s'intéresser à mon information ? ». Dans le monde entier, les sondages disponibles sur les connaissances et intérêts du public confirment que ceux-ci sont largement inférieurs à ce que pourraient escompter les professionnels (voir Labasse, 2000 pour une synthèse). Ainsi, aux Etats-Unis, " An analysis of public attentiveness to more than 500 news stories over the last ten years confirms that the American public pays relatively little attention to many of the serious news stories of the day. " (Parker \& Deane, 1997). Le problème qui se pose à la presse n'est pas foncièrement différent de celui que rencontre le rédacteur tentant, au sein d'une entreprise ou d'une institution, de donner à 
son texte une chance d'être lu : informer n'est pas seulement fournir, c'est aussi séduire, montrer d'abord que la question mérite qu'on s'y intéresse.

Depuis au moins Dewey (1913), et sans doute bien avant, il est admis que l'intérêt n'est pas intrinsèque aux faits à communiquer mais réside dans la relation qui s'instaure entre ceux-ci et celui qui en prend connaissance. D'un point de vue psychologique et pédagogique, la théorie de l'intérêt est loin d'être aussi développée qu'on pourrait le souhaiter, mais quelques repères permettent de mieux en percevoir les enjeux. La littérature cognitive s'accorde globalement à distinguer au moins deux catégories :

Some things are inherently interesting, like sex and violence. Anything that has to do with the self, in fact, is in this category. (...) The other class of interesting things are those that are neither too familiar nor too strange (...) Those are the things that arouse our curiosity, without necessarily being linked to our own self. (Kintsch, 1997, pp. 418-419)

Plus détaillé, le modèle de Schank (1979) articule les facteurs d'intérêt d'un texte en quatre grandes familles (Martins, 1993, p. 99) :

- Les facteurs « absolus », qui touchent des points tels que la mort, la maladie, le pouvoir la sexualité, etc. ;

- Les facteurs « personnels », qui concernent l'existence propre du lecteur ;

- Les facteurs inattendus, qui provoquent une surprise, diffèrent de ce que le lecteur aurait pu prévoir ;

- "Enfin la dernière catégorie est une catégorie particulière : ce sont des informations qui ne sont pas intéressantes en elles-mêmes mais qui combinées entre elles présentent de l'intérêt.».

Dans notre perspective, on peut donc retenir dans tous les cas une distinction fondamentale : soit l'intérêt « va de soi », c'est-à-dire qu'il repose sur une relation spontanée entre les données et le lecteur (soit primale, soit surdéterminée par les intérêts propres de celui-ci), soit il ne va pas de soi, et la relation doit alors être construite. L'un des éléments essentiels de cette construction est la " problématisation » : le fait de susciter un déséquilibre cognitif, une attente. Ce point est surtout familier aux didacticiens ; "l'art de poser des questions provocatrices est au moins aussi important que celui qui consiste à donner des réponses claires »(Bruner, 1996, p. 158), mais ses implications sont beaucoup plus générales (voir Ram, 1990). En fait, elles sont mêmes plus importantes dans la vie en général que dans la salle de classe, où l'attention est de toute façon contrainte (par l'obligation de présence, la perspective des examens et le maître lui-même). Du reste, le principe même de problème est au cœur de la rhétorique. Pour Meyer (1999), c'est-là une de ses caractéristiques fondamentales : « elle traite de questions, de la problématicité, de l'énigme. » (p. 242). S'il n'y a pas de 
question à résoudre, il ne peut y avoir de débat, et le discours est inutile en soi. Cet auteur étend même le raisonnement au langage en général :

Parler, c'est soulever une question. Ecrire aussi. C'est pourquoi on ne parle pas de ce qui va de soi [...] le langage est utilisé pour attirer l'attention sur une question c'est ce qu'on appelle la communication* ${ }^{*}$ à propos de laquelle on pourrait être en désaccord, ou tout simplement, à côté de laquelle on pourrait passer. (Meyer, 1999, pp. 73-74; * en ital. dans l'original)

Or l'art du lead en $5 \mathrm{~W}$, comme l'art de la phrase courte et des mots simples, est précisément celui de fournir des réponses claires à des questions (Qui ? Quoi ?, etc.) sans se demander si lecteur se les pose. Fondamentalement, il constitue plus une technique d'expression des faits qu'une technique de communication des faits. II n'est donc pas bon ou mauvais en soi, et son utilité ne dépend pas nécessairement de la longueur du texte à produire. Elle dépend essentiellement du caractère "spontané » ou " à construire » de l'intérêt du sujet. Dans le premier cas - par exemple une catastrophe provoquant des centaines de morts - il va sans dire que la présentation des faits se suffit à elle-même et demande peu de compétence rédactionnelle. C'est le type même de textes que parviennent déjà à produire de façon relativement convaincante des prototypes de systèmes rédactionnels automatiques - des journalistes-robots, en quelque sorte - tels que Newsblaster développé par une équipe de l'Université de Columbia. Mais pour la majorité des thèmes importants de l'actualité - politiques, économiques, scientifiques, etc. - un tel traitement, loin d'être " efficace " est au contraire remarquablement inadéquat, parce que les « faits " à présenter ne répondent à aucune attente cognitive et se trouvent ainsi abandonnés à eux-mêmes.

On perçoit ce handicap en comparant par exemple l'attractivité de deux attaques portant sur le même sujet, mais dont l'une, passive, est centrée sur les faits [A], et l'autre, construite, est centrée sur le problème $[\mathrm{B}]$ :

[A] Une équipe de l'Institut de physique nucléaire de Lyon va installer cet hivers près de Modane (Savoie), un instrument destiné à étudier les particules massives à faible interaction (WIMPS). L'appareil, un détecteur à très basse température, sera placé dans une cavité communiquant avec le tunnel du Fréjus. (Information brute, non publ.)

[B] L'Univers nous cache quelque chose. Quelque chose d'énorme, dont nous ne savons strictement rien, si ce n'est, précisément, que ça nous est caché. Les physiciens ont en effet l'irritante conviction que toute la matière connue ne représente qu'une faible fraction de la masse du cosmos, et que la nature du reste leur échappe totalement. (Isotopes nº, septembre 1994 p. 8) 
Le lead en $5 \mathrm{~W}+\mathrm{H}$ et le plan rétropyramidal posent, du reste, d'autres problèmes lorsqu'on les utilise mécaniquement, même dans le cas des sujets à fort « intérêt spontané». Une construction revenant à ajouter à une information de base des précisions successives conduit par exemple souvent à produire des textes absurdement redondants, comme dans cet extrait d'une dépêche d'agence de presse (soulignée par nous) :

\section{L'A320 de Gulf Air a effectué une approche à une "vitesse excessive"}

30 août $2000-20 h 48$

MANAMA (AFP) - L'Airbus A320 de Gulf Air, qui s'est abîmé le 23 août en mer à son atterrissage à Bahrein, a effectué une approche finale à une "vitesse excessive" ne pouvant pas lui permettre de se poser, a annoncé mercredi un responsable de l'Aviation civile de Bahrein.

"La vitesse de l'Airbus de Gulf Air au moment de son approche finale vers l'aéroport de Bahrein était excessive", a déclaré le sous-secrétaire du ministère des Transports pour les affaires de l'Aviation civile Ibrahim Al-Hamer, lors d'une conférence de presse à Manama.

"Cette vitesse est excessive pour un avion de ce type en phase d'approche finale avant de se poser (...) c'est pour cela que la tour de contrôle (ATC) a conseillé au pilote de remonter de 600 pieds à 2.500 pieds d'altitude (180 à près de 800 mètres) pour tenter une deuxième approche", a poursuivi M. Hamar.

Il a toutefois souligné que "les raisons du crash ne sont pas encore connues, mais d'après les résultats préliminaires de l'enquête, la vitesse de l'approche finale de l'A320 était de 270 noeuds (environ $490 \mathrm{~km} / \mathrm{h}$ ), donc plus élevée que la vitesse maximale pour une approche finale". (...)

II n'est donc pas surprenant qu'au moins deux études de grande ampleur (Denton, 1993 ; Readership institute, 2001), menées auprès de lecteurs de presse, aient montré que les textes employant ce procédé étaient globalement moins bien reçus et retenus que d'autres formes d'exposition (narrative, explicative, etc.). Ce qui est surprenant est que tant d'enseignants en rédaction, d'auteurs de manuels et de responsables éditoriaux ne s'en soient pas avisés de longue date. En somme, la logique qui soustend les $5 \mathrm{~W}$ et la pyramide, si elle est intégrée sans une claire conscience de ses limites, conduit à attribuer systématiquement au lecteur une responsabilité qui n'est pas seulement la sienne, mais tout autant celle du rédacteur. La responsabilité de percevoir 
la valeur de l'information et même celle d'évaluer la longueur que celle-ci mérite (en interrompant sa lecture lorsqu'il estime avoir « suffisamment » d'information) ${ }^{11}$.

Le lead en $5 \mathrm{~W}$ et la pyramide inversée possèdent une justification dans divers cas, liés soit au fond (informations factuelles simples et répondant à une attente spontanée) ou à la forme (nouvelles brèves, dépêches d'agence). II peuvent être d'excellents outils, au même titre qu'un tournevis cruciforme ou un spectromètre de masse, mais, comme eux, ils ne sont utiles qu'à celui Qui sait à Quoi, Quand, Où, Pourquoi et Comment les utiliser. On a eu raison, sans doute, de conserver de la rhétorique la séquence Quis, Quid, Ubi, Quibus auxiliis, Cur, Quomodo, Quando, mais peut être aurait-il fallu examiner tout l'héritage :

Nous, c'est le goût des autres qui doit régler notre langage, et le plus souvent il nous faut parler devant des hommes sans culture aucune, et, dans tous les cas, ignorants de l'art dont je viens de parler; si nous ne savons pas les attirer par le plaisir, les entraîner par la force, et parfois les troubler par l'émotion, nous ne pourrons faire triompher même la justice et la vérité. (Quintilien, op. cit., p. 275).

En résumé, l'examen des « savoirs » rédactionnels de base que sont le lead et la pyramide inversée conduit a relever de multiples antinomies :

- entre leur origine conventionnellement admise et leur genèse réelle ;

- entre la simplification (structuration des idées) que visent les $5 \mathrm{~W}$ et la surcharge cognitive qu'ils peuvent provoquer ;

- entre le « message essentiel » qu'ils visent à cerner et le « scénario circonstanciel » qu'ils tendent à forcer ;

- entre le sens attaché au terme " pyramide » et la représentation mentale qu'il suscite ;

- entre l'image de l'une et l'autre de ces notions hors du champ du journalisme et les distances que prennent avec elles beaucoup de professionnels des médias ;

- entre leur justification par le contexte informationnel (surabondance des informations) et leur efficacité dans ce contexte (abdication du rédacteur) ;

- entre leurs justifications «psychologiques » (accessibilité) et leur limite fondamentale (intérêt) selon ce même critère ;

- Et plus globalement, entre deux prescriptions également fréquentes : " soyez intéressant ${ }^{2}$ » et « allez droit aux faits ».

Ces observations donnent une idée de la distance qu'il reste à parcourir aux techniques rédactionnelles pour devenir une véritable méthodologie de la rédaction,

\footnotetext{
${ }^{11}$ Hormis les cas particuliers, doit-on considérer qu'un texte qu'un lecteur abandonne au milieu est un bon texte (puisqu'il est construit pour cela) ou un mauvais texte (puisque le lecteur ne va pas jusqu'au bout)?

12 Parfois exprimé sous une forme plus impérieuse : "Si votre première phrase ne fait pas mouche, inutile de chercher la seconde ».
} 
épistémologiquement et praxéologiquement digne de ce nom. Elles permettent également de penser qu'il existe des liens entre des questions ou des niveaux qui sont rarement reliés entre eux : une dimension sociale (les citoyens connaissent mal l'économie, le droit, les sciences, etc.) une dimension corporative (les journaux perdent des lecteurs) et une dimension purement technique (la méthodologie de la rédaction). Cette dernière ne saurait évidemment résoudre à elle seule les autres, mais son importance n'en est pas moins évidente. 


\section{References}

ADIT - AGENCE POUR LA DIFFUSION DE L'INFORMATION TECHNOLOGIQUE (1999). Cahier spécial : Rédiger avec pertinence.Technologies Internationales, 52, 521.

ANTOINE, F., et al. (1995). Ecrire au quotidien ; Pratiques du journalisme. Lyon : Chronique Sociale.

BALLE, F. (1987). Et si la presse n'existait pas. Paris : Editions Jean-Claude Lattès.

BARRASS, R. (1978). Scientists must write. Londres : Chapman \& Hall.

BÉNICHOUX, R. (Dir). (1985). Guide pratique de la communication scientifique. Paris : Ed. G. Lachurié.

BERROU, J.-P. (1991). Bien écrire en affaires. Paris : Dunod.

BOCLÉ, C., \& PARIOT, C. (1997). La puissance de I 'écrit. Saâcy : La Bohème.

BONNAMOUR, J.-Y. (1997). Guide pratique de l'écrit. Lyon : Chronique sociale.

BRUNER, J.S. (1996). L'Education, entrée dans la culture. Paris : Retz.

BRUNI, F. \& ALFIERI, G. (1997). Manuale di scrittura professionale. Bologne : Zanichelli.

CARRIÈRE, C. \& SCHREIBER, C. (2000). Création de pages web. Polycopié du cours "Utilisation pédagogique du multimédia" (version pdf). Grenoble : IUFM.

COLLECTIF. (1992). Bien écrire et parler juste. Paris : Sélection du Reader's Digest.

CRÊTE, J., \& IMBOT, L. (1996). Comprendre et communiquer la science. Bruxelles : De Boeck.

CTA - CENTRE TECHNIQUE DE COOPÉRATION AGRICOLE ET RURALE. (2000). Communication et développement : L'art de se faire comprendre. Spore, 86, 4-5.

DEWEY, J. (1913). Interest and Effort in Education. Boston, MA : Houghton-Mifflin.

ERRICO, M. et al. (1997). The evolution of the summary news lead. Media History Monographs, 1 (1). Athens $(\mathrm{OH})$ : Ohio University.

FAURE, D. (1998). Concevoir, illustrer et diffuser vos messages. St. Laurent du Mesnil : Maxima.

FLESCH, R. (1949).The art of readable writing. New York : Macmillan. 
FLORIO, R. (1984). L'écriture de presse. Lille : Trimédia /ESJ.

GRAÑA-GONZÁLES, M. (1930). La Escuela de periodismo. Madrid : C.I.A.P.

GUNNING, R. (1968). The technique of clear writing. New York : MacGraw Hill.

HARTMAN, W. (1968). Journalism. River Forest (IL) : Laidlaw Brothers.

HERVOUET, L (1979). Ecrire pour son lecteur . Lille : Trimédia /ESJ.

HORACE. (1996). Epître aux Pisons. In R. Bouchet (Trad.), Satires et épîtres (pp. 6979). Paris : Hatier.

KINTSCH, W. (1998). Comprehension : a paradigm for cognition. Cambridge (MA) : Cambridge University Press.

LABASSE, B. (2000). Information et citoyenneté : Des fissures dans la "société du savoir ». Lyon : Mission prospective de la Communauté Urbaine de Lyon.

LASSWELL, H. D. (1948). The Structure and Function of Communication in Society. In L. Bryson (ed.), The Communication of Ideas. New York :Harper and Brothers (pp. 3751). Reprint, in W. Schramm \& D. F. Roberts (Eds.) : The process and effects of mass communication (pp. 84-89). University of Illinois Press, 1971.

LORENZ, A.L., \& VIVIAN, J. (1996). News : Reporting and Writing. New York : Allyn \& Bacon.

MARTIN-LAGARDETTE, J.-L.(1994). Ecrire, informer, convaincre ; les secrets de l'écriture journalistique. Paris :Syros.

MARTINS, D. (1993). Les facteurs affectifs dans la compréhension et la mémorisation des textes. Paris: Presses universitaires de France.

MEYER, M. (Ed.), (1999). Histoire de la rhétorique des grecs à nos jours. Paris : Librairie générale française.

MINDICH, D. (1998). Just the Facts : How "Objectivity" Came to Define American Journalism. New York: New York University Press.

MOURIQUAND, J. (1997). L'écriture journalistique. Paris : Presses universitaires de France.

PARKER, K., \& DEANE, C. (1997). Ten Years of the Pew News Interest Index. Washington: The Pew Research Center for the People and the Press.

PERELMAN, C. (1997). L'empire rhétorique; rhétorique et argumentation. Paris : Vrin. 
PIERRON, C., \& Pierron, O. (1996). Cinq leçons pour rédiger efficacement. Alleur (B) : Marabout.

PORUSH, D. (1995). A Short Guide to Writing About Science. New York : HarperCollins.

QUINTILIEN. Institution oratoire, Livres IV-VI. (Trad. H. Bornecque). Paris : Garnier, s.d. (ca.1933).

READERSHIP INSTITUTE. (2001). The Value of Feature-style Writing. Evanston, IL : Readership Institute.

STEPHENS, M. (2002). A j-school manifesto. Zoned for Debate. Retrieved from http://journalism.nyu.edu/publishing/archives/debate/forum.1.essay.stephens.html

$\mathrm{BIO}$

Bertrand Labasse est professeur agrégé au Département de français de l'Université d'Ottawa. II était précédemment directeur scientifique du centre de recherchedéveloppement des éditeurs de presse français. Ses travaux portent sur les déterminants psychologiques et sociaux de l'information et de la communication écrite, ainsi que sur les processus de traitement et de réception des informations complexes. II est l'auteur de plusieurs ouvrages sur la méthodologie, la didactique et l'épistémologie de ces domaines. 